\title{
Une collection de livres
}

Jacques Le Brun

\section{(2) OpenEdition}

Journals

Édition électronique

URL : http://journals.openedition.org/rbnu/1028

DOl : $10.4000 /$ rbnu. 1028

ISSN : 2679-6104

\section{Éditeur}

Bibliothèque nationale et universitaire de Strasbourg

\section{Édition imprimée}

Date de publication : 1 mai 2017

Pagination : 112-121

ISBN : 9782859230661

ISSN : 2109-2761

\section{Référence électronique}

Jacques Le Brun, "Une collection de livres », La Revue de la BNU [En ligne], 15 | 2017, mis en ligne le 01 novembre 2019, consulté le 12 décembre 2020. URL : http://journals.openedition.org/rbnu/1028 ;

DOI : https://doi.org/10.4000/rbnu.1028

\section{(c) $\odot \Theta(\odot)$}

La Revue de la BNU est mise à disposition selon les termes de la Licence Creative Commons Attribution - Pas d'Utilisation Commerciale - Partage dans les Mêmes Conditions 4.0 International. 
VARIA

\section{Une collection de livres}

PAR JACQUES LE BRUN
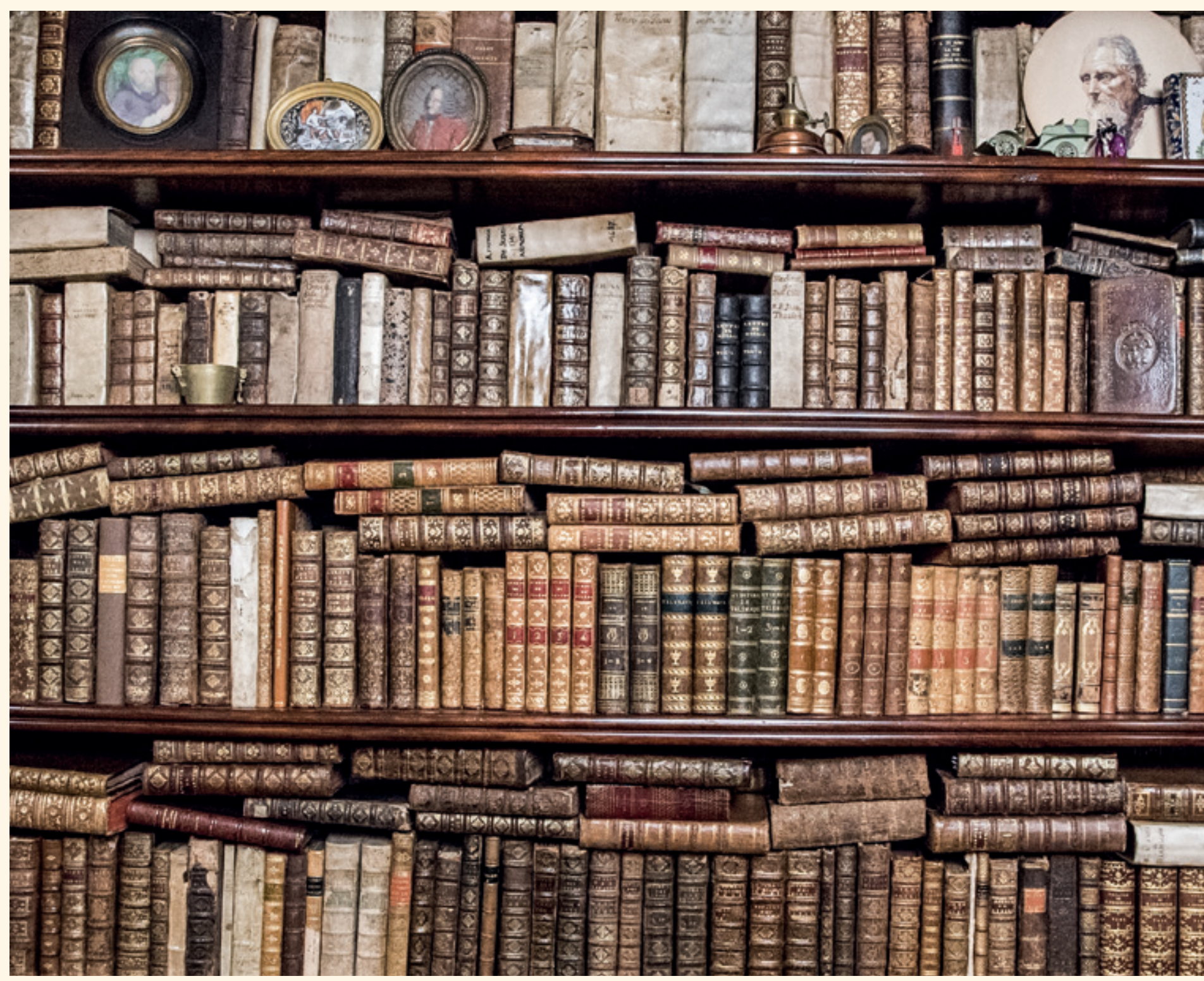

La bibliothèque de Jacques Le Brun (vue partielle) 
Quand on parle de collection ou de collectionneur, chacun a en l'esprit un ensemble d'objets, certains rares ou très rares, rassemblés en une unité générique (des timbres-poste, des faïences d'Urbino, des livres, etc.) par le désir et les soins d'un collectionneur qui a choisi l'objet de cette collection ou dont l'objet s'est un jour imposé à lui, qui a eu la compétence pour savoir quelle pièce acquérir et laquelle mépriser ou qui a confié ce soin et cette compétence à un spécialiste, à un historien d'art, à un bibliothécaire ou à un archiviste, enfin qui a eu les moyens d'entretenir cette collection, de la développer, de l'étudier, de connaître l'exacte nature, voire la valeur, des pièces rassemblées. Ainsi les grandes collections de livres et manuscrits constituées par des personnages connus, par exemple le duc d'Aumale ou Sir Thomas Phillipps et cent autres au $19^{\mathrm{e}}$ siècle, un Gabriel Hanotaux, un Jacques Doucet, un Henri Mondor, un Henri de Rothschild, et bien d'autres encore au $20^{\mathrm{e}}$, chacun ayant à son service des bibliothécaires de grande compétence.

Or il ne s'agit de rien de tel pour l'ensemble de livres appelé « collection Jacques Le Brun » dans le catalogue de l'exposition Fénelon et son double ${ }^{1}$, ensemble de livres constitué autour des sciences et de la littérature religieuses de l'âge classique, du $16^{\mathrm{e}}$ au $18^{\mathrm{e}}$ siècles, où l'on rencontre les noms d'auteurs bien connus comme Fénelon, Bossuet, Mme Guyon, Richard Simon, Pierre-Daniel Huet, Pierre Nicole et les auteurs moins connus de livres de spiritualité ascétique et mystique, de biographies de religieuses, d'écrits se rapportant aux grands débats théologiques et historiques de ce temps-là.

La première question que l'on pourrait se poser et qu'avec le recul du temps, de plus d'une soixantaine d'années, je ne peux pas ne pas me poser, c'est la façon dont a pu survenir et s'imposer chez un jeune étudiant se destinant à un travail universitaire l'idée et le désir de rassembler des éditions anciennes des auteurs sur lesquels il travaillait, éditions qu'il utilisait et manipulait chaque jour à la Bibliothèque nationale pour préparer un Diplôme d'études supérieures vers 1953.

On pourrait se donner d'emblée la facile explication par ce que l'on pourrait appeler l'hérédité ou l'influence familiale. Il est vrai que plusieurs ancêtres ou collatéraux avaient possédé ou possédaient alors d'importantes bibliothèques et avaient eux-mêmes publié un certain nombre de livres, si bien que c'est pour ainsi dire sous forme de «livres », possédés ou écrits et publiés, que, dans une généalogie, ils prenaient forme et visage dans l'imagination de cet enfant. À cela s'ajoutait le fait que dans la demeure de ses parents, la pièce qui lui paraissait centrale dans son isolement était la bibliothèque où des milliers de livres offraient à ses lectures enfantines et adolescentes des ressources inépuisables, essentiellement des livres du $19^{\mathrm{e}}$ et du premier $20^{\mathrm{e}}$ siècles, mais aussi tous les classiques des siècles anciens. En tout cas, de ces importantes bibliothèques, seuls des fragments sont parvenus jusqu'ici et ont pu constituer l'embryon de la collection dont quelques pièces ont été exposées à la Bibliothèque nationale et universitaire de Strasbourg, et ce n'est que quelques années après les débuts de ce rassemblement de livres appelé « collection » que quelques-uns de ces livres sont arrivés par héritage. Cela ne signifie pas que certains apports ne furent pas décisifs. Je ne prendrai qu'un exemple, une collection d'Ana, ces recueils de citations, de conversations et de fragments d'écrivains ou d'humanistes dont la vogue s'étendit des années 1650 au début du $19^{\mathrm{e}}$ siècle. Un arrière-grand-père ${ }^{2}$ avait, au sein d'une immense bibliothèque, constitué une collection d'une centaine d'Ana; ayant hérité celle-ci, il a été possible, sans trop de difficultés, par des achats d'un ou deux volumes par an à des prix modestes, de doubler le nombre de ces livres et, évidemment sans atteindre l'ampleur de la collection dont Aude en 1910 avait établi la bibliographie ${ }^{3}$, d'en constituer un ensemble significatif.

Cependant je crois qu'évoquer cette archéologie personnelle n'est pas l'essentiel. Constituer une collection de ce genre n'est pas avant tout mettre ses pas dans ceux de ses prédécesseurs. Selon une explication plus convaincante, la collection semble répondre à des préoccupations précises : ne serait-ce pas, pour quelqu'un qui commence à travailler dans un champ du savoir, se constituer un ensemble permettant d'avoir sous la main, disponibles jour et nuit, ses instruments de travail, gain de temps, et plus grande facilité dans l'usage et la comparaison des livres ? Il y aurait donc ici une dimension utilitaire, avoir chez soi une Bibliothèque nationale en miniature !

Je m'arrêterai peu à ces évidences. Et pourtant, même de ce point de vue utilitaire, le rassemblement d'un fonds d'éditions anciennes est intéressant pour des raisons qui ne sont pas tout de suite accessibles à qui n'a pas eu cette expérience. Il y a là, et nous atteignons peut-être une autre dimension de la bibliothèque, quelque chose qui touche à la nature même d'un livre, cet objet unique dont, malgré les apparences d'une uniforme multiplicité, chaque exemplaire n'est jamais semblable aux autres. Je reviendrai sur ce point. Mais très vite, en travaillant sur les livres de la Bibliothèque nationale et en acquérant des 
exemplaires des $16^{\mathrm{e}}, 17^{\mathrm{e}}$ et $18^{\mathrm{e}}$ siècles sur lesquels on travaille aussi, on remarque des signes souvent peu visibles au premier regard : ex-libris anciens, pages interfoliées, cartons, soulignements, notes marginales, cachets de bibliothèques anciennes ou récentes, etc. Tel exemplaire, semblable en apparence à celui qu'on avait entre les mains à la $\mathrm{BN}$, s'est révélé unique et fournit des renseignements que n'apporte pas ce dernier (qui d'ailleurs en apporte d'autres, non moins intéressants). Donnons un exemple de cette unicité et des renseignements qu'elle peut nous apporter : des éditions de Jean de Bernières, de Benoît de Canfield ou $\mathrm{du}$ père Surin, mystiques du $17^{\mathrm{e}}$ siècle, tous mis à l'index lors de l'offensive antimystique des années 1680-1700, portent une signature et une date, ou une mention de bibliothèque, la signature d'une religieuse, carmélite ou visitandine, l'ex-libris d'un couvent, une date du milieu $\mathrm{du} 18^{\mathrm{e}}$ siècle, montrant qu'en ces années des religieuses continuaient à lire, à recopier et à méditer ces œuvres mystiques du siècle précédent et par ailleurs ne tenaient nul compte des index et des interdictions des autorités ecclésiastiques. On peut tirer un grand nombre de remarques de ce genre d'exemplaires de ces livres de spiritualité achetés à vil prix, qui m'ont apporté nombre d'éléments exploités dans ma thèse complémentaire en 1970 sur Les opuscules spirituels de Bossuet et leur diffusion dans les milieux religieux de Nancy au $18^{\mathrm{e}}$ siècle ${ }^{4}$.

C'est d'ailleurs ce caractère du livre, constituer l'unité d'une série et en même temps être un objet unique porteur d'informations, que révèlent à un examen approfondi les conditions d'impression à l'âge classique, sans parler des fréquentes et remarquables qualités esthétiques d'exemplaires par ailleurs modestes. Ainsi, nous nous apercevons par exemple que les œuvres polémiques de Fénelon, lors de la querelle du pur amour, n'ont pas été imprimées chacune chez un seul imprimeur, mais qu'elles l'ont été simultanément chez plusieurs, comportant pour le même texte des différences de caractères, de mise en page, de papier et de filigrane, renseignements précieux qui nous révèlent des stratégies éditoriales, une hâte à répondre aux objections, des réseaux s'étendant sur plusieurs villes. L'examen d'un seul exemplaire d'une bibliothèque, cette dernière fût-elle prestigieuse, ne suffit pas pour une étude précise des conditions de développement d'une querelle théologique au $17^{\mathrm{e}}$ siècle. Mais nous entrons ici dans le domaine de la bibliographie matérielle, si utile pour connaître l'histoire des œuvres. Quand réaliserat-on cette bibliographie matérielle des éditions des Aventures de Télémaque des seules années 1699-1701?

\section{Les religieuses $[\ldots]$ ne tenaient nul compte des index et des interdictions des autorités ecclésiastiques}

Tâche immense, à peine ébauchée depuis le travail pionnier d'Albert Cahen ${ }^{5}$.

Et ici, celui qui a empiriquement et au hasard des découvertes rassemblé des ouvrages anciens concernant ses objets d'étude, s'aperçoit que la grande entreprise, si utile, si indispensable même, de numérisation des livres anciens des grandes bibliothèques, risque paradoxalement, chez certains utilisateurs trop pressés, de susciter une idée tout à fait inexacte de ce qu'est un livre. Disons, de façon un peu provocatrice, qu'un livre n'est pas le support d'une information, ou, de façon moins brutale, n'est pas seulement le support d'une information. Lire un livre ancien, mais peut-être aussi lire sur écran un livre d'aujourd'hui, risque d'en donner une idée inexacte. Un livre est ce qu'on pourrait appeler une « chose», un « objet ", en donnant à ces mots leur sens le plus fort qui n'est pas seulement celui d'un instrument en vue d'une fonction, la communication d'un message. Un livre est un objet, c'est-à-dire un ensemble de matières, de formes, de déformations, et susceptible de différents usages, de même que sa réalisation n'a pas seulement répondu à des exigences techniques. En tant qu'objet, il est essentiellement unique, même si aujourd'hui il est plus facilement reproductible qu'autrefois, circonstance historique qui est une des sources de l'illusion. Il est unique, non seulement dans sa fabrication, mais aussi unique dans les usages qui en sont faits et dans l'investissement affectif dont il est le support, parfois même aux limites du fétichisme. Mais pourquoi pas? Ne condamnons pas cette conception 
Copie manuscrite d'un livre publié en 1670, mis à l'index en 1690, copie effectuée par une religieuse du $18^{\mathrm{e}}$ siècle (coll. J. Le Brun).

Meffire jean de bernieres Louvigning Eocuier Conseillar Jun Roy et trésorier de france. a cäen, morten oleur des Sainteté le 3:may 1639 . aagé de 54 . ans.

Celivere est a liusage de Joent marie Régis quila trans crit avec permiftion de Ses fupientes en novembre 1441 .

Sonnée par La Jour Masice. Jelphine le thast ispo.a La Joent Masie Mág Jeleinu Richarg carmelite.
Les acures fpirituelles de monsieser de Bernieres Louvigni, or.

Conduite affuré pour Ceux qui tendent a la perfection.

Seconde partice Contenant les lettres qui font voir la pratique des maximes. $\sim \sim \sim \cdots \sim \sim \sim$ a paris,
cher claude Cramoisy, rie st. jacques, proche le collige a u plestis, au Sacrifice J'abel. M.DC.LXX. avec privilége of appodations.

115 

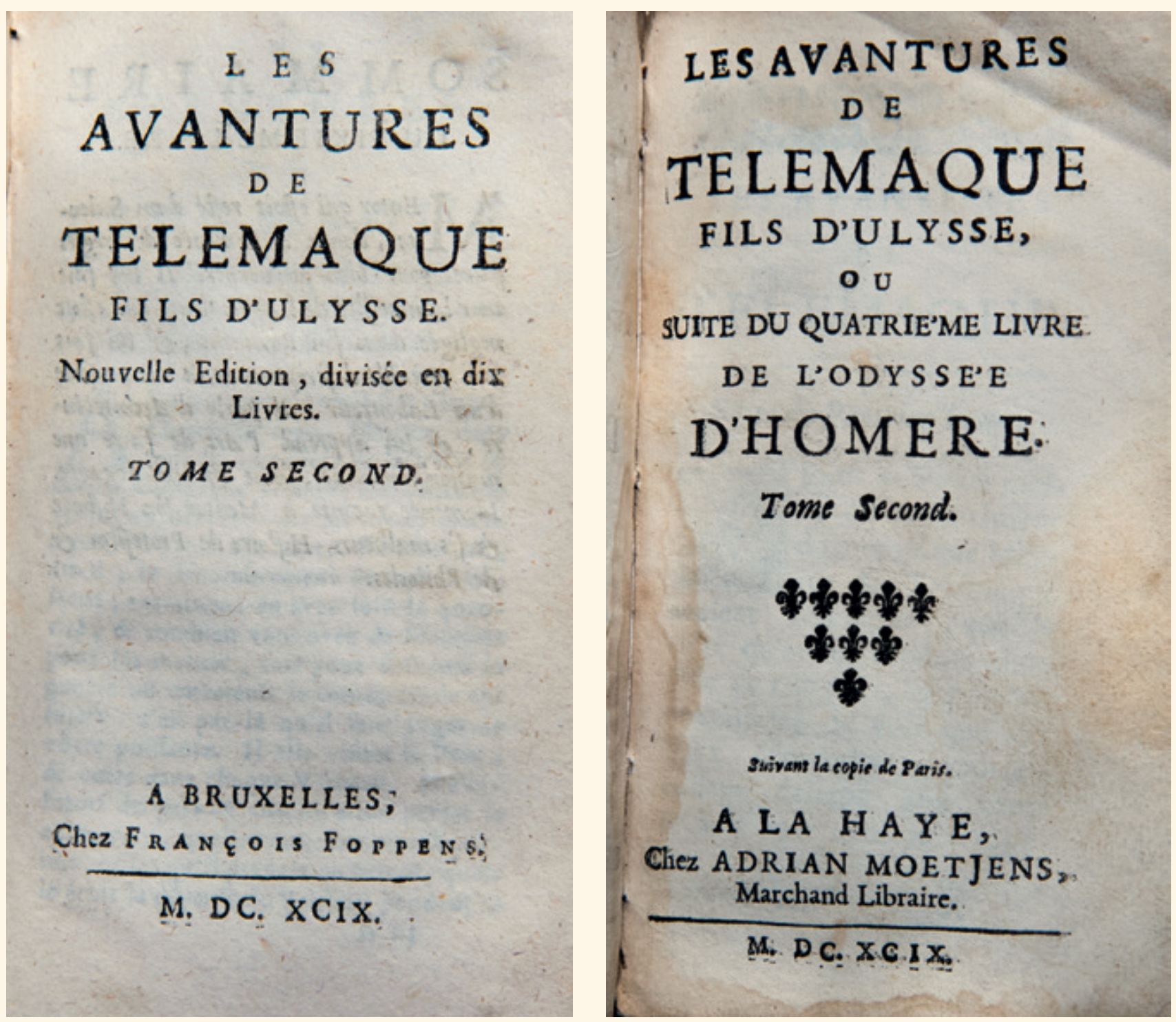

Deux des nombreuses éditions des Aventures de Télémaque parues clandestinement en 1699,

l'année de la première édition (coll. J. Le Brun). 
fétichiste de l'objet-livre. Inversement, s'il est objet par l'usage qui en est fait, ce caractère d'objet se retourne et modifie la signification qu'il comporte, le caractère de l'information qu'il contient. Un exemple pris encore une fois à l'œuvre de Fénelon : les Aventures de Télémaque aux innombrables éditions différentes par leur format, les caractères d'imprimerie, les illustrations, les langues dans lesquelles elles sont traduites, sont devenues, du fait des usages divers qu'elles ont pu susciter, le point aveugle de rencontre de ces innombrables usages et la référence, peut-être imaginaire malgré la matérialité des exemplaires, des innombrables significations que les lecteurs en trois siècles y ont trouvées ou à partir d'elles ont créées : œuvre réactionnaire, progressiste, dévote, éclairée, philosophique ou encore ésotérique. Du fait d'être objets uniques, ces livres intitulés «Aventures de Télémaque » sont devenus supports de significations et d'affects souvent contradictoires ; la multiplicité des exemplaires semble brouiller la signification alors qu'elle modifie, ou même crée, cette signification. Et cette multiplicité, si elle peut refléter le désir accumulateur, voire fétichiste, du collectionneur, est aussi le reflet de l'inscription du livre dans une histoire, de la constitution d'une série (par exemple les éditions du Télémaque au $18^{\mathrm{e}}$ siècle), donc l'élément d'une généalogie, tout en étant également représentative d'une autre série, celle des exemplaires publiés en nombre plus ou moins grand en même temps que cet exemplaire que nous avons entre les mains. Comme l'écrit Pierre Legendre, « un document n'est pas un déchet informationnel, mais un témoin qui nous parle au-delà de ce qu'il énonce : il n'existe qu'en rapport avec une généalogie de textes, il n'est donc jamais isolable ${ }^{6}$. D'où une justification du maniement de tel ou tel exemplaire. Rien là de la conception contemporaine de l'information, la transmission sans reste ni déformation d'un message entre émetteur et récepteur, conception dont les techniques modernes, par ailleurs si utiles et si fécondes, si indispensables, peuvent favoriser l'empire.

La question que nous soulevons ici n'a nulle part été mieux exposée et analysée que dans l'essai que publia en 1936 Walter Benjamin et qui s'intitule L'œuvre d'art à l'ère de sa reproductibilité technique ${ }^{7}$, essai qui depuis longtemps m'a paru présenter le meilleur instrument pour penser les mutations que les techniques modernes ont opérées dans le champ de l'art et de la culture. Ce que Benjamin y écrit de l'œuvre d'art peut s'appliquer au livre, dans la mesure où le livre, comme nous venons de le voir, possède lui aussi le caractère d'unicité de l'objet, de la « chose », de l'œuvre d'art. «Unicité », c'est le mot qu'emploie ici Walter Benjamin lorsqu'il écrit : «à la plus parfaite reproduction il manque toujours quelque chose, l'ici et le maintenant de l'œuvre d'art l'unicité de sa présence au lieu où elle se trouve »; et il ajoute : "C'est à cette présence unique pourtant et à elle seule que se trouve liée toute son histoire ${ }^{8}$. En introduisant l' " histoire » comme dimension de l'unicité et de la présence, ce que dans cet essai il appelle aussi l'aura, Walter Benjamin nous apporte de précieux éléments sur la nature particulière du livre à la fois multiple et unique. Selon lui, l'histoire de l'œuvre, qui est liée à " son pouvoir de témoignage historique " ${ }^{9}$, donc « l'autorité de la chose » - ce sont ses mots -, ce sont non seulement les " altérations matérielles", mais aussi la « succession de ses possesseurs ", et nous reconnaissons ici ce que nous tentions d'exposer il y a quelques instants en nous interrogeant sur le rapport entre telle édition d'un livre et tel exemplaire de ce même livre, deux régimes d'historicité, l'un celui du collectif et de la reproduction, l'autre celui de l'individuel et de la suite des lecteurs à travers les siècles et les traces de leurs lectures successives, ce que Walter Benjamin appelle aussi le « domaine de la tradition $" ~{ }^{10}$. Ainsi, s'il est vrai que le livre, non seulement dans sa multiplicité, mais aussi dans la singularité de chaque exemplaire, peut être un instrument de travail, porteur d'un message historique, sociologique ou encore politique, donc susceptible de la part de son possesseur et utilisateur d'un investissement intellectuel ou informationnel, il est aussi ce que Walter Benjamin désignait des deux mots, à prendre en un sens plein, un « objet " (il parle de « l'aura d'un objet naturel ») ou " une chose ». Et là le rapport à l'objet, à la chose est d'un autre ordre que celui du travail intellectuel, que celui de la science, fût-elle science des textes, science $\mathrm{du}$ livre. Pour tenter de rendre sensible cette autre forme d'investissement de cet objet qu'est le livre, je prendrai, rapidement, la question de plus haut et, au risque de surprendre, je remonterai à saint Augustin et à sa distinction fondatrice, dans son traité $D e$ diversis quaestionibus, entre jouir et utiliser (dans son latin «frui » et « uti »), distinction sur laquelle j'ai pu naguère présenter un travail à propos de la question de l'amour pur sur laquelle les auteurs des livres présentés dans l'exposition Fénelon et son double ont longuement et vivement débattu ${ }^{11}$.

Est-ce qu'aimer c'est désirer une récompense, est-ce chercher une jouissance désintéressée qui n'est accompagnée d'aucune préoccupation d'utilité ? Utiliser est ainsi opposé à jouir dans la mesure où jouir c'est 
Deux pages d'un exemplaire annoté

par Phélipeaux, trésorier de l'église

de Meaux, proche de Bossuet,

de l'Explication des maximes des saints (1697)

de Fénelon (coll. J. Le Brun).

Maximes des Saints. $\quad 221$ Elles ont une paix \& une joye pleine d'innocence. Elles prennent avec fimplicité \& fans he-por/ryil fitation les foulagemens d'ef-dragrion prit \& de corps qui leur font veritablement neceflaires, comme elles les confeilleroient à leur prochain. Elles parlent d'elles mêmes fans en juger pofitivement, mais par pure obéillance \& pour le vrai befoin fuivant que les chofes leur paroiffent dans le moment même. Elles en parlent alors fimplement en bien ou en mal comme elles parleroient d'autruy, fans aucun attachement ni à ce qui leur paroît, ni à la bonne opinion que leurs paroles les plus fimples \& les plus modeftes pourroient donner d'elles, \& reconnoiffant toûjours avec une humble joye T iij
Maximes des Saints. 119 I'efprit. C'éroit dăs de tels actes que faint Antoine mettoit l'Oraifon $\mathrm{Ia}$ plus parfaite, quand il difoit; LOraifon neft point Caniert encore parfaite, quand le solitai- conf, 9 . re connoift qu'il fait Orailon.

Parler ainfi, c'eft parler fuivant l'experience des Saints fans bleffer la rigueur du dogme catholique. C'eft même parler des operations de l'ame conformément aux idées de zous les bons Philofophes.

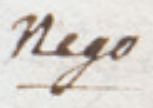

\section{FAUX.}

II $n^{y} y$ a point de veritables actes que ceux qui font réflechis \& qu'on fent ou qu'on ap-celaesl uray perçoit. Dés qu'on n'en faie plus de cette façon, il eft vrai de dire qu'on n'en faic pensee. parceleomente eslaperace. parcelomene ou morins danes instam quclle ex siste quorque.

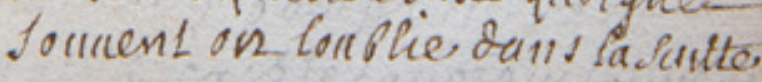




\section{«Rien de moins libre qu'un collectionneur, ça se voit à l'œil nu»}

se porter vers une " chose ", une res - nous pourrions dire un « objet » - pour elle-même. L'objet-livre sera donc autre chose qu'un instrument de connaissance, d'acquisition, de savoir ou de plaisir. Il sera envisagé pour lui-même. Cet objet attire pour lui-même, et ceux qui en ont fait l'expérience peuvent témoigner que ce n'est pas fondamentalement pour telle qualité esthétique, bibliophilique ou culturelle qu'il attire. Comme l'écrit Krzysztof Pomian dans un livre essentiel sur la collection aux $17^{\mathrm{e}}$ et $18^{\mathrm{e}}$ siècles, celle-ci appelle « une 'consommation' qui se fait par l'intermédiaire du simple regard et n'entraîne donc pas de destruction physique ${ }^{12}$. Et l'auteur met en cause l'« utilité » à propos de la collection : «On ne peut sans commettre un abus de langage étendre la notion d'utilité au point d'en attribuer une à des objets qui ne font que s'offrir aux regards ». La remarque qui porte sur l'œuvre d'art peut et doit aussi s'appliquer à cet « objet » qu'est le livre, malgré l'évidente (et envahissante ?) utilité qu'il présente. Car cet objet suscite par lui-même un désir qui est un acte ; saint Augustin l'avait bien montré dans son analyse de la jouissance. Mais ce désir et cette jouissance ne sont pas arrêtés par la possession, et c'est ici qu'intervient ce qu'on appelle collectionner, répéter, dans une quête, en son essence infinie, cet acte dont aucune possession n'étanche le désir. Et cela malgré la conviction que jamais la collection ne sera " complète ». Comme le notait finement en 2014, dans un essai consacré à la collection, Gérard Wajcman ${ }^{13}$ : «Toute collection est [...], dans son principe, un acte délibéré et libre, de pure liberté, de pur désir ", mais une liberté qui comporte en elle-même sa négation. L'essayiste ajoute que cet acte est « accompli sous la contrainte, la férule tyrannique de l'objet. Rien de moins libre qu'un collectionneur, ça se voit à l'œil nu ». Acte contradictoire dont la liberté est de s'abandonner en une perte totale de liberté, acte que cette contradiction rend proche de celui de l'adoration; dans le texte de 1936 que j'évoquais tout à l'heure, Walter Benjamin parlait du « collectionneur qui ressemble toujours un peu à un adorateur de fétiches ", avec l'expérience de celui qui collectionna livres d'enfants et jouets, qui parla merveilleusement de ces collections et assista aussi, de son exil, à la perte de ces dernières ${ }^{14}$.

Contradictoire dans son acte, libre et serve, la collection de ces " choses " qui sont des " objets " est aussi contradictoire dans ce qu'elle suscite. L'objet, en son sens étymologique, objectum, objicere, montrer, pour ainsi dire jeter aux yeux, est voué à se montrer, même si le collectionneur cache sa collection. Il la cache peutêtre pour ne pas révéler à travers elle l'objet de son désir, voire qu'il est animé du désir de l'objet et de la chose. Comme l'écrivait encore Gérard Wajcman dans l'essai auquel je faisais allusion à l'instant : « Une collection est un ensemble organisé d'objets qui montre, qui se montre et qu'on montre. À quelqu'un. Le regard fait partie de la collection ", et il ajoute que " toute collection fait place, ou sa place, au regard $»^{15}$.

Pour instructive qu'elle soit, la distinction augustinienne que j'évoquais et qui nous conduisait à nous intéresser à cette jouissance de l'objet dont la collection nous donnait un exemple convaincant, doit être interrogée. En effet la distinction, qui semble radicale, entre l'utilisation (intéressée, visant l'acquisition d'un savoir, d'une information) et la jouissance (désintéressée, acte pur du désir de la chose) est, par saint Augustin lui-même et par les nombreux philosophes et théologiens qui l'ont commentée, accompagnée d'une réflexion sur les points de passage entre l'une et l'autre de ces polarités : quel excès et débordement de l'utilité ne glisse pas à la jouissance? quelle jouissance n'entraîne une involontaire acquisition, puis un usage qui se révèle d'extrême utilité ? Et ici, après avoir paru m'en écarter, mais sans au fond l'avoir totalement perdu de vue, je reviendrai à mon objet, au livre, sous la forme du livre ancien des $16^{\mathrm{e}}-18^{\mathrm{e}}$ siècles dans le champ de la littérature spirituelle et de dévotion. Tout se passe en effet comme si l'on ne pouvait penser l'un des termes de l'opposition que par rapport à l'autre. Il existe des collectionneurs qui ne sont pas des collectionneurs purs, et il existe des historiens pour qui le livre n'est pas un simple support d'information ; oserai-je me situer dans cette catégorie pour ainsi dire hybride ? Ce livre-ci ou celui-là peut être à la fois un instrument de connaissance, irremplaçable aussi à ce titre, et en même temps, au moment même où il apporte d'irremplaçables renseignements sur tel débat théologique ou telle question historique, cet " objet » support d'un investissement affectif du fait de sa 
singularité ; mais cet investissement n'est pas lui-même dépourvu de raison, même si l'attachement à l'objet peut dépasser la simple raison : souvenir du lieu et du moment de l'achat, évocation de la bibliothèque dont il provient, méditation sur le destin de cette bibliothèque ou sur ce que purent être la vie, les pensées, les émotions de tel possesseur qui a mis son nom ou son ex-libris, au $18^{\mathrm{e}}$ ou au $20^{\mathrm{e}}$ siècle, sur la première page, rêverie sur ce que pourra être son destin futur, etc. Il se crée un attachement fait de tout un réseau de pensées et d'affects qui constitue à proprement parler ce que Walter Benjamin appelait l'aura de l'objet; et tout cela au moment où l'objet apporte des informations historiques, littéraires, philosophiques rigoureuses.

Si le livre des $17^{\mathrm{e}}$ et $18^{\mathrm{e}}$ siècles est pour nous document historique, support d'un travail d'une irremplaçable utilité, et s'il est en même temps objet d'un désir, ce livre pourrait n'être que le mirage d'un rêve inaccessible. En effet, la réalité impose ici ses conditions. Ces conditions sont doubles, mais l'une et l'autre déterminent les possibilités d'acquisition des livres ; d'abord l'existence d'un marché, d'une offre, selon lesquels des livres peuvent être achetés, et d'autre part les conditions de ce marché, des prix, qui ne soient pas des obstacles infranchissables à leur acquisition. Posons cette banalité : il n'y a pas de collection de livres s'il n'y a pas de possibilité matérielle (une offre et des prix) de les acquérir.

Or toute période de l'histoire ne réalise pas ces deux conditions. Ne parlons pas ici des éditions précieuses, des exemplaires somptueux avec des provenances exceptionnelles : ces livres sont réservés à toute époque à des collectionneurs pour qui ce sont rarement des instruments de travail, et qui ont les moyens de les acquérir. Dans le domaine qui est le nôtre (l'histoire de la théologie, de la spiritualité, les sciences religieuses), deux moments de l'histoire ont offert des conditions exceptionnelles, permettant de se constituer facilement une bibliothèque. Le premier de ces moments, déjà lointain, ce sont les lendemains de la Révolution française, alors que les considérables bibliothèques religieuses, de couvents, d'abbayes ou de séminaires furent nationalisées et enrichirent les bibliothèques publiques, non sans qu'une bonne partie de leurs livres échappent à ce transfert et soient mis sur le marché : dans notre domaine, les bibliothécaires, souvent gallicans et jansénisants, négligèrent souvent les livres de spiritualité, qui se trouvèrent ainsi mis en vente.

Plus près de nous, le milieu du $20^{\mathrm{e}}$ siècle, les années 1950-1970, vit la fermeture d'un grand nombre de couvents, de séminaires, de maisons religieuses, à travers toute l'Europe. Une extraordinaire quantité de livres anciens dans le domaine religieux se retrouva alors sur le marché et, du fait de l'abondance et du nombre limité d'amateurs, était proposée à des prix accessibles, parfois dérisoires. Se trouver à ce moment permettait de se constituer un irremplaçable instrument de travail et d'accéder à un désir. Ces conditions n'étaient ni durables ni reproductibles ; une fois les sources épuisées, l'offre devint rare et les prix de moins en moins accessibles. Mais une bibliothèque, riche sans être prestigieuse, avait pu en ces années se constituer et associer en cette chose qu'est le livre ancien un instrument de travail et l'objet d'un désir.

\section{NOTES}

1- Fénelon et son double, catalogue réalisé sous la direction de François-Xavier Cuche et Julien Gueslin, Strasbourg, Bibliothèque nationale et universitaire, 2015

2- Louis Brunet (1827-1913), auteur d'un recueil de textes spirituels des $17^{\mathrm{e}}-19^{\mathrm{e}}$ siècles, Officium sanctissimi cordis $D$. N. Jesu-Christi, « offert par l'auteur », 1904 [" seconde édition revue et très augmentée ", 1909].

3- A. F. Aude, Bibliographie critique et raisonnée des ANA, Paris, Darragon, 1910

4- Jacques Le Brun, Les opuscules spirituels de Bossuet. Recherches sur la tradition nancéienne, Nancy, « Annales de l'Est. Mémoires, $\mathrm{n}^{\circ} 38 », 1970$

5- Fénelon, Les aventures de Télémaque, nouvelle édition publiée [...] par Albert Cahen, Coll. des grands écrivains de la France, 2 vol., Paris, Hachette, 1920

6- Pierre Legendre, Fantômes de l'État en France, Paris, Fayard, 2015, p. 186

7- Dans Walter Benjamin, Euvres, coll. Folio, Paris, Gallimard, 2000, t. III, p. 67-113 et dernière version, ibid., p. 269-316

8- Ibid., p. 273

9- Ibid., p. 275

10- Ibid., p. 276

11- Jacques Le Brun, Le pur amour de Platon à Lacan, Paris, Seuil, 2002, p. 65-88

12- Krzysztof Pomian, Collectionneurs, amateurs et curieux. Paris, Venise : XVI ${ }^{\text {ème}}-X V I I I^{\text {ème }}$ siècles, Paris, Gallimard 1987, p. 12

13- Gérard Wajcman, Collection, suivi de L'avarice, Éd. Nous, 2014, p. 37

14- Le « collectionneur qui ressemble toujours un peu à un adorateur de fétiches et qui par la possession même de l'œuvre d'art participe à son pouvoir culturel », Walter Benjamin, op. cit., p. 280, n. 2. Voir le texte si attachant de Walter Benjamin, Je déballe ma bibliothèque. Une pratique de la collection, Paris, Rivages Poche, 2000.

15- Gérard Wajcman, op. cit., p. 46-47 


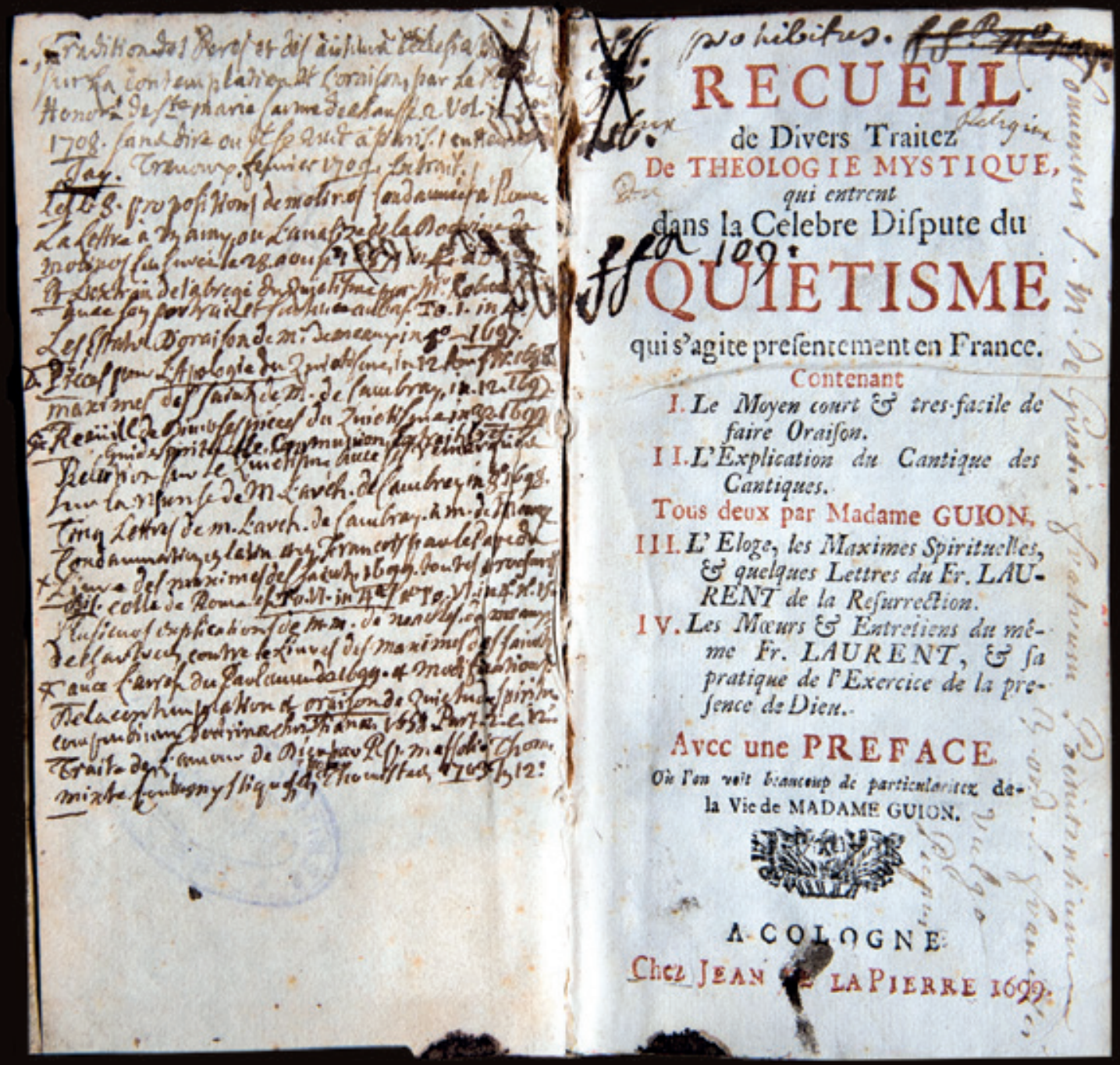

Recueil de textes en particulier de Mme Guyon,

figurant à l'index depuis 1689 (d'où la mention « prohibitus »)

et provenant de la bibliothèque des « Frères Pénitents du Tiers-Ordre de S. François couramment appelés Picpus », à Paris (coll. J. Le Brun). 